\title{
Effects of 12-Month Valsartan Therapy on Glycation and Oxidative Stress Markers in Type 2 Diabetic Subjects With Hypertension
}

\author{
Naoko KomiYA, ${ }^{1}$ MD, Hiroshi Hirose, ${ }^{1,2}$ MD, Yoshifumi SAISHO, ${ }^{1}$ MD, \\ Ikuo SAITO, ${ }^{1,2} \mathrm{MD}$, and Hiroshi ITOH, ${ }^{1} \mathrm{MD}$
}

\section{SUMMARY}

Although it has been reported that angiotensin II receptor blockers inhibited the formation and accumulation of advanced glycation endproducts (AGEs) in vitro and in vivo, whether they can do so clinically is not clear. We investigated the effects of 12-month valsartan therapy on various markers of inflammation, glycation, and oxidation in type 2 diabetic subjects with hypertension.

We started $40 \mathrm{mg}$ /day valsartan treatment in 15 type 2 diabetic patients with hypertension. In 6 patients, the dose of valsartan was increased to $80 \mathrm{mg}$ /day after 6 months and maintained until 12 months. Metabolic parameters including BMI and serum high molecular weight (HMW)-adiponectin, high-sensitivity C-reactive protein (hs-CRP) as an inflammation marker, AGEs, paraoxonase activity, platelet-activating factor (PAF)acetylhydrolase activity, and urine 8-isoprostane levels were measured at baseline and after 6 and 12 months of treatment. Urine microalbumin level and carotid artery intimamedia thickness (IMT) were also measured.

Even after valsartan therapy, the blood pressure levels of the patients were not decreased significantly. Serum AGEs and urine 8-isoprostane levels decreased at both 6 and 12 months $(P<0.05$ for both), although other metabolic and oxidative markers were unchanged. Though urine microalbumin levels tended to be decreased after 6 and 12 months of valsartan treatment, the changes were not significant. Mean IMT at 12 months was not changed from the baseline value. In conclusion, the findings suggest that treatment with valsartan, even at a low dose, may ameliorate some glycation and oxidative stress markers independently of an effect on blood pressure in hypertensive type 2 diabetic subjects. (Int Heart J 2008; 49: 681-689)

Key words: Angiotensin II receptor blocker, Advanced glycation endproducts, Oxidative stress markers, Type 2 diabetes mellitus

THE prevalence of type 2 diabetes mellitus is increasing worldwide ${ }^{1)}$ as well as in Japan, ${ }^{2)}$ predisposing the patients to a high risk of developing diabetic microan-

From the ${ }^{1}$ Department of Internal Medicine, and ${ }^{2}$ Health Center, Keio University School of Medicine, Tokyo, Japan.

Address for correspondence: Hiroshi Hirose, MD, Department of Internal Medicine, Keio University School of Medicine, 35 Shinanomachi, Shinjuku-ku, Tokyo 160-8582, Japan.

This study was supported in part by research grants (to H.H.) from Keio University, Tokyo.

Received for publication July 24, 2008.

Revised and accepted September 1, 2008. 
giopathy and atherosclerotic vascular diseases (ischemic heart disease, cerebrovascular disease, and peripheral artery disease). It is well-known that when diabetic subjects become hypertensive, the risks of developing microangiopathy and macroangiopathy are much higher than in nondiabetic subjects. ${ }^{3,4)}$

As for nephropathy, it has been shown that an angiotensin II receptor blocker (ARB), valsartan, has renoprotective effects in patients with type 2 diabetes mellitus, independently of blood pressure. ${ }^{5}$ However, the detailed mechanisms of such effects remain unclear. Advanced glycation endproducts (AGEs) have been focused on as one of the causal candidates, because AGEs are formed by glycation and oxidation in the Maillard reaction both in vitro and in vivo, and their levels are increased in diabetic subjects. ${ }^{6,7)}$ Also, an association between AGEs and diabetic microangiopathy has been reported, and intervention to reduce AGEs is considered an important strategy in treating diabetic nephropathy. ${ }^{8)}$

Miyata, et al reported that ARBs or angiotensin-converting enzyme inhibitors (ACE-Is) decreased AGE formation in vitro via radical scavenging and transition metal chelation, ${ }^{9)}$ and it has been shown that some ARBs reduced renal AGE accumulation and proteinuria in diabetic rodents in vivo ${ }^{10-12)}$ to a degree similar to that of an ACE-I. ${ }^{13)}$ Sebekova, et al reported that treatment with the ACE-I ramipril for 2 months significantly decreased the fluorescent AGE level in 12 subjects with nondiabetic nephropathy. ${ }^{14)}$

In the present study, we investigated the effects of valsartan treatment in Japanese type 2 diabetic subjects for up to 12 months, on various markers of glycation and oxidation as well as metabolic parameters. We also measured serum high molecular weight (HMW)-adiponectin and high-sensitivity C-reactive protein (hs-CRP) as metabolic and inflammatory markers, urine microalbumin level as a marker of nephropathy, and carotid artery intima-media thickness (IMT) to assess the degree of atherosclerosis.

\section{METHODS}

Subjects: Fifteen type 2 diabetic outpatients with hypertension being treated by the Department of Internal Medicine, Keio University Hospital, Tokyo, participated in this study. The patients included 14 men and 1 woman. As shown in Table I, the mean age was $63.2 \pm 7.8$ (SD) years and the mean duration of diabetes was $8.1 \pm 3.8$ years. Body mass index (BMI) was $24.8 \pm 4.0 \mathrm{~kg} / \mathrm{m}^{2}$ and the fasting plasma glucose was $151 \pm 15 \mathrm{mg} / \mathrm{dL}$. We recruited subjects whose diet therapy or medication had not been changed for at least 3 months and whose glycemic control was relatively stable for this period (mean hemoglobin $A_{1 C} 6.7 \pm 0.6 \%$ ). 
Table I. Subject Profiles at Baseline

\begin{tabular}{lcc}
\hline & Range \\
\hline$n$ (male/female) & $14 / 1$ & \\
Age (years) & $63.2 \pm 7.8$ & $46-74$ \\
Duration of diabetes (years) & $8.1 \pm 3.8$ & $2-16$ \\
Height $(\mathrm{cm})$ & $165.7 \pm 4.7$ & $159.0-177.0$ \\
Weight $(\mathrm{kg})$ & $68.2 \pm 11.4$ & $48.0-90.0$ \\
Body mass index (kg/m ${ }^{2}$ ) & $24.8 \pm 4.0$ & $18.1-33.9$ \\
Systolic blood pressure (mmHg) & $149 \pm 15$ & $122-182$ \\
Diastolic blood pressure (mmHg) & $86 \pm 11$ & $67-109$ \\
Fasting plasma glucose (mg/dL) & $151 \pm 15$ & $118-177$ \\
Hemoglobin A $(\%)$ & $6.7 \pm 0.6$ & $5.6-7.6$ \\
Microvascular complications & & \\
$\quad$ (retinopathy, nephropathy, neuropathy) & $4,2,2$ & \\
Macrovascular complications & & \\
$\quad$ (coronary, cerebral, peripheral) & $0,2,0$ & \\
Current smoking & $10(66.7 \%)$ & \\
Therapy for type 2 diabetes & & \\
$\quad$ (diet only, oral hypoglycemic agent, insulin) & $0,14,1$ & \\
Statin therapy & $3(20.0 \%)$ & \\
\hline
\end{tabular}

Data are $n$ or mean $\pm \mathrm{SD}$.

Five patients were being treated with a calcium channel blocker and one with a beta-blocker at baseline, but none with an ACE-I, ARB, or diuretic. Antihypertensive agents being taken by the patients other than valsartan were not changed during the study. All patients were treated with oral hypoglycemic agents, but one with insulin. Ten patients were current smokers, and three had been treated with an HMG-CoA reductase inhibitor (Table I).

The present study was conducted according to the principles expressed in the Declaration of Helsinki, 1964 and the Declaration of Tokyo, 1975, as revised in 1983. Informed consent was obtained from each subject after full explanation of the purpose, nature, and risk of all procedures used. The protocol was approved by the ethical review committee of the Department of Internal Medicine, School of Medicine, Keio University, Tokyo. Some of the study subjects also participated in one of our previous studies. ${ }^{15)}$ However, we extended the follow-up period from 6 months to 12 months, and we examined serum HMW-adiponectin and hs-CRP levels, as well as measuring carotid artery mean IMT as a marker of atherosclerosis.

Treatment with $40 \mathrm{mg}$ /day of valsartan in the morning was started. In 6 out of 15 patients, the dose of valsartan was increased to $80 \mathrm{mg} /$ day (40 mg tablet twice daily) after 6 months and maintained at that level until 12 months.

Measurements: Various metabolic parameters, namely serum AGEs, paraoxo- 
nase activity, platelet-activating factor acetylhydrolase (PAF-AH) activity, and urine 8-isoprostane levels were measured at baseline and 6 and 12 months after initiation of treatment.

BMI was calculated as weight in $\mathrm{kg}$ divided by height in meters squared. Systolic blood pressure and diastolic blood pressure were measured with subjects in the sitting position after resting for at least 5 minutes. The blood samples were collected around $9 \mathrm{AM}$ in the morning after an overnight fast. Plasma glucose, serum low-density lipoprotein (LDL)-cholesterol, high-density lipoprotein (HDL)-cholesterol, triglycerides, and uric acid levels were assayed by routine automated laboratory methods, as described previously. ${ }^{16-18)}$

HMW-adiponectin was measured using a commercially available kit (HMW Adiponectin ELISA Kit, Fujirebio Inc., Tokyo). This ELISA system does not need a denaturing step, and the monoclonal antibody (IH7) is reported to react specifically with the HMW form of adiponectin. ${ }^{19)}$ The dilution curve was parallel to the standard curve. Intra- and interassay coefficients of variation (CV) were 2.4-3.0\% and 4.2-5.1\%, respectively. Serum hs-CRP level was measured by nephelometry, using a latex particle-enhanced immunoassay (N Latex CRP II, Dade Behring, Tokyo) with both intra- and interassay $\mathrm{CV}$ of $<5.0 \%$. The assay could detect $0.005 \mathrm{mg} / \mathrm{dL}$ of CRP.

Serum paraoxonase activity was measured by colorimetric assay, as previously described, ${ }^{20)}$ and PAF-AH activity was also measured by colorimetric assay (PAF acetylhydrolase assay kit, Cayman, Ann Arbor, MI, USA). Serum AGEs were measured by ELISA as previously described, ${ }^{7)}$ and the intra- and interassay CV of this ELISA system were 4.8-10.2\% and 3.5-6.2\%, respectively. Urine-8isoprostane was measured by ELISA (8-isoprostane EIA kit, Cayman). Creatinine clearance ( $\mathrm{Ccr}$ ) was calculated using the Cockcroft-Gault equation.

Assessment of common carotid artery IMT: Ultrasonography of the carotid arteries was performed, as described previously, ${ }^{21,22)}$ using an echotomographic system (LOGIC S6 and LOGIC 7, GE Yokogawa Medical System Inc., Tokyo) with a linear transducer (mid-frequency range of 7-12 MHz). Scanning of the extracranial carotid arteries in the neck was performed bilaterally in 3 different longitudinal projections (anterior-oblique, lateral, and posterior-oblique) and in the transverse projections. This allowed the common carotid artery, carotid bulb, and internal carotid artery to be scanned bilaterally. All of the images were photographed.

The common carotid artery IMT was defined as the distance from the leading edge of the first echographic line to the leading edge of the second echographic line on the scans, with the first line representing the collagen-containing upper layer of the tunica adventia. In each longitudinal projection, the site of the greatest IMT thickness was detected by scanning along the vessel from the com- 
mon carotid artery to the internal carotid artery. Three measurements of the IMT on both sides were performed at the site of greatest thickness and 2 other points ( $1 \mathrm{~cm}$ proximal and $1 \mathrm{~cm}$ distal to this site) for each patient. The highest value of the 6 averaged values of IMT ( 3 from the right side and 3 from the left side) was used as the representative mean IMT for each patient. A few specialized physicians performed all the scans and IMT measurements.

Statistical analysis: Statistical analyses were performed using the SPSS ${ }^{\circledR}$ program for Windows (version 15.0-J, SPSS Japan Inc., Tokyo). $P$ values $<0.05$ were considered to denote statistical significance. The Wilcoxon signed-rank test was used to compare the serum HMW-adiponectin level and carotid artery IMT before and 12 months after valsartan treatment. Analysis of variance (ANOVA) followed by the Dunnett test was used to compare the other parameters before to 6 and 12 months after valsartan treatment. Because serum triglycerides, HMW adiponectin, hs-CRP and AGEs levels, and urine 8-isoprostane level were normally distributed after logarithmic transformation, we used logarithms of these data in the analyses.

\section{RESULTS}

The change in each parameter before to after the treatment is shown in Tables II and III. Even after valsartan treatment (40-80 mg/day), the blood pressure level of the patients did not change during the study. In 6 patients, to whom $40 \mathrm{mg} /$ day of valsartan was added in the evening from 6 months to 12 months, blood pressure levels were still not changed at both 6 and 12 months (from 148 /

Table II. Changes in Metabolic Parameters Between Before and After Valsartan Treatment (40-80 mg/day) in 15 Type 2 Diabetic Subjects

\begin{tabular}{lcccc}
\hline & Baseline & 6 months & 12 months & $P$ \\
\hline Body mass index $\left(\mathrm{kg} / \mathrm{m}^{2}\right)$ & $24.8 \pm 4.0$ & $25.8 \pm 3.3$ & $25.3 \pm 3.7$ & $\mathrm{NS}$ \\
Systolic blood pressure $(\mathrm{mmHg})$ & $149 \pm 15$ & $145 \pm 22$ & $146 \pm 20$ & $\mathrm{NS}$ \\
Diastolic blood pressure $(\mathrm{mmHg})$ & $86 \pm 11$ & $84 \pm 12$ & $86 \pm 13$ & $\mathrm{NS}$ \\
Hemoglobin $\mathrm{A}_{1 \mathrm{C}}(\%)$ & $6.7 \pm 0.6$ & $6.8 \pm 0.9$ & $7.0 \pm 1.0$ & $\mathrm{NS}$ \\
LDL-cholesterol $(\mathrm{mg} / \mathrm{dL})$ & $133 \pm 19$ & $132 \pm 21$ & $123 \pm 20$ & $\mathrm{NS}$ \\
HDL-cholesterol $(\mathrm{mg} / \mathrm{dL})$ & $52 \pm 11$ & $50 \pm 11$ & $49 \pm 10$ & $\mathrm{NS}$ \\
Triglycerides $(\mathrm{mg} / \mathrm{dL})$ & $137 \pm 69$ & $145 \pm 64$ & $154 \pm 71$ & $\mathrm{NS}$ \\
Uric acid $(\mathrm{mg} / \mathrm{dL})$ & $5.9 \pm 1.0$ & $6.1 \pm 0.9$ & $6.1 \pm 1.2$ & $\mathrm{NS}$ \\
HMW adiponectin $(\mu \mathrm{g} / \mathrm{mL})$ & $5.6 \pm 2.3$ & - & $5.7 \pm 2.0$ & $\mathrm{NS}$ \\
hs-CRP $(\mathrm{mg} / \mathrm{dL})$ & $0.81 \pm 0.39$ & $0.91 \pm 0.59$ & $1.11 \pm 0.89$ & $\mathrm{NS}$ \\
\hline
\end{tabular}

Values are the mean \pm SD. ${ }^{* *} P<0.01$ versus baseline by Dunnett test. NS indicates not significant; LDL, low-density lipoprotein; HDL, high-density lipoprotein; HMW, high molecular weight; and hs-CRP, high sensitivity C-reactive protein. 
Table III. Changes in Markers of Glycation, Oxidation, and Diabetic Complications Between Before and After Valsartan Treatment (40-80 mg/day) in 15 Type 2 Diabetic Subjects

\begin{tabular}{lcccc}
\hline & Baseline & 6 months & 12 months & $P$ \\
\hline AGEs (unit/L) & $2.71 \pm 0.62$ & $2.23 \pm 0.36 *$ & $2.29 \pm 0.28 *$ & 0.021 \\
Paraoxonase activity (unit/L) & $239 \pm 68$ & $230 \pm 74$ & $233 \pm 60$ & $\mathrm{NS}$ \\
PAF-AH (mmol/L/minute) & $20.1 \pm 5.1$ & $18.9 \pm 5.7$ & $17.3 \pm 5.7$ & $\mathrm{NS}$ \\
Urine 8-isoprostane (ng/gCr) & $347 \pm 215$ & $190 \pm 116^{*}$ & $205 \pm 122^{*}$ & 0.025 \\
Creatinine (mg/dL) & $0.86 \pm 0.14$ & $0.87 \pm 0.14$ & $0.89 \pm 0.16$ & $\mathrm{NS}$ \\
Calculated Ccr (mL/minute) & $86.7 \pm 26.7$ & $88.4 \pm 27.2$ & $84.7 \pm 24.2$ & $\mathrm{NS}$ \\
Urine microalbumin (mg/gCr) & $167 \pm 258$ & $114 \pm 218$ & $128 \pm 247$ & $\mathrm{NS}$ \\
Carotid artery mean IMT (mm) & $0.81 \pm 0.18$ & - & $0.86 \pm 0.20$ & $\mathrm{NS}$ \\
\hline
\end{tabular}

Values are the mean \pm SD. $* P<0.05$ versus baseline by Dunnett test. NS indicates not significant; AGEs, advanced glycation endproducts; PAF-AH, platelet-activating factor acetylhydrolase; Ccr, creatinine clearance; and IMT, intima-media thickness.

$88 \mathrm{mmHg}$ to 158 / 91 and $160 / 95 \mathrm{mmHg}$, respectively, $P>0.6$ ). BMI and HbA1c level increased slightly during the study, though the increases were not significant. Serum paraoxonase activity and PAF-AH levels were also unchanged. In contrast, serum AGE levels significantly decreased at both 6 and 12 months (from $2.71 \pm 0.62$ unit/L to $2.23 \pm 0.36$ and $2.29 \pm 0.28$ unit/L, respectively, $P<$ 0.05 for both) after valsartan treatment (Table III). The urine 8 -isoprostane level also decreased significantly at both 6 and 12 months (from $347 \pm 215 \mathrm{ng} / \mathrm{gCr}$ to $190 \pm 116$ and $205 \pm 122 \mathrm{ng} / \mathrm{gCr}$, respectively, $P<0.05$ for both).

Neither the serum creatinine level nor calculated Ccr changed during the study. The urine microalbumin level was slightly decreased after 6 and 12 months of valsartan treatment (from $167 \pm 258 \mathrm{mg} / \mathrm{gCr}$ to $114 \pm 218$ and $128 \pm 247 \mathrm{mg}$ / $\mathrm{gCr}$, respectively), however, the changes were not significant. Carotid artery mean IMT was not changed at 12 months compared with the baseline value (from $0.81 \pm 0.18 \mathrm{~mm}$ to $0.86 \pm 0.20 \mathrm{~mm}, P=0.22$ ).

There were no significant correlations between the changes in AGEs or urine 8-isoprostane levels with the changes in urine microalbumin levels, calculated Ccr, or IMT.

\section{Discussion}

JNC-7 ${ }^{23)}$ and JSH 2004 ${ }^{24)}$ both recommended that the target blood pressure in patients with type 2 diabetes mellitus should be below 130/80 $\mathrm{mmHg}$. Although we increased the dose of valsartan to $80 \mathrm{mg}$ /day from 6 months to 12 months in 6 out of 15 patients, mean blood pressure was not decreased. One of the reasons why valsartan treatment in this study could not decrease mean blood pressure might have been the increase in BMI during the study period, or that the 
relatively low-dose valsartan treatment might have been insufficient in diabetic subjects to achieve the target blood pressure in the morning before taking the medication.

It has been reported that the ARBs olmesartan ${ }^{9-11)}$ and candesartan ${ }^{12)}$ inhibited the formation and accumulation of AGEs in vitro ${ }^{9)}$ and in diabetic animal models. ${ }^{10-12)}$ However, whether they can do so clinically is not clear. In our study, valsartan treatment significantly decreased the urine 8-isoprostane level as well as serum AGEs. However, other markers did not change during the study. Although the detailed mechanism(s) of the effects of valsartan on AGEs in vivo are unclear, the antioxidative effect of valsartan might be one of the mechanisms. Recently, Monacelli, et al described the effects of valsartan on AGEs and oxidative stress markers in 12 type 2 diabetic subjects. ${ }^{25}$ ) They found that 3 to 6 -month valsartan treatment (80-160 mg/day) significantly decreased blood pressures, plasma pentosidine and carboxymethyhl-lysine (CML) concentrations, both of which are major components of AGEs, and urine 15-F2t-isoprostanes levels.

In the present study, we have shown that relatively low-dose valsartan therapy (40-80 mg/day) decreased serum AGE and urine 8-isoprostane levels, and also slightly reduced the urine microalbumin level for an extended period of 12 months. It is noteworthy that these beneficial effects were observed even if the blood pressure did not decrease significantly. Although the lack of a significant reduction in blood pressure was unexpected, these findings suggest valsartan therapy has some kind of blood pressure-independent effect. Although the change in AGE level was not associated with the change in urine microalbumin level or calculated Ccr in our study, a significant positive correlation between renal pentosidine content and proteinuria has been reported in a rat model. ${ }^{10,11)}$ Further longitudinal studies are needed to clarify whether the lowering of serum AGE levels affects the clinical outcome of diabetic microangiopathy.

Accumulating evidence suggests that oxidative stress may play a crucial role in the development of atherosclerosis. ${ }^{26-28)}$ For example, it has been reported that circulating levels of 8-isoprostane were an independent risk factor associated with coronary artery disease in a Chinese population (odds ratio $2.47, P<$ $0.001),{ }^{26)}$ and were associated with peripheral artery disease. ${ }^{27,28)}$ In this study, we have shown that valsartan therapy decreased urine 8-isoprostane levels up to 12 months in type 2 diabetic subjects with hypertension, despite the fact that the blood pressure levels were unchanged. Although carotid artery mean IMT did not decrease after 12 months of treatment in this study, there is a possibility that valsartan treatment of a longer period might inhibit the progression of atherosclerosis via suppression of oxidative stress in type 2 diabetic subjects. Further longitudinal studies of much longer duration with different sex, ethnicity, and/or ARB would facilitate the understanding of this important issue. 
In conclusion, the findings of the present study suggest that treatment with valsartan, even at a low dose, may ameliorate some glycation and oxidative stress markers independently of an effect on blood pressure in hypertensive type 2 diabetic subjects.

Disclosure statement: Fujirebio Inc., Tokyo (formerly Chugai Diagnostic Science Inc., Tokyo) and Dr. Hirose have a partial patent concerning the HMW-adiponectin measurement kit. Dr. Saito has received a research grant from Novartis Pharma K.K., Tokyo.

\section{REFERENCES}

1. Reusens B, Ozanne SE, Remacle C. Fetal determinants of type 2 diabetes. Curr Drug Targets 2007; 8: 935-41. (Review)

2. Nakano T, Ito H. Epidemiology of diabetes mellitus in old age in Japan. Diabetes Res Clin Pract 2007; 77: S7681.

3. Expert Committee on the Diagnosis and Clasification of Diabetes Mellitus. American Diabetes Association: clinical practice recommendations 2002. Diabetes Care 2002; 25: S1-147.

4. Nakamura Y, Saitoh S, Takagi S, et al. Impact of abnormal glucose tolerance, hypertension and other risk factors on coronary artery disease. Circ J 2007; 71: 20-5.

5. Viberti G, Wheeldon NM; MicroAlbuminuria Reduction With VALsartan (MARVAL) Study Investigators. Microalbuminuria reduction with valsartan in patients with type 2 diabetes mellitus: a blood pressure-independent effect. Circulation 2002; 106: 672-8.

6. Brownlee M. Advanced protein glycosylation in diabetes and aging. Annu Rev Med 1995; 46: 223-34. (Review)

7. Ono Y, Aoki S, Ohnishi K, Yasuda T, Kawano K, Tsukada Y. Increased serum levels of advanced glycation end-products and diabetic complication. Diabetes Res Clin Pract 1998; 41: 131-7.

8. Jerums G, Panagiotopoulos S, Forbes J, Osicka T, Cooper M. Evolving concepts in advanced glycation, diabetic nephropathy, and diabetic vascular disease. Arch Biochem Biophys 2003; 419: 55-62. (Review)

9. Miyata T, van Ypersele de Strihou C, Ueda Y, et al. Angiotensin II receptor antagonists and angiotensin-converting enzyme inhibitors lower in vitro the formation of advanced glycation end products: biochemical mechanisms. J Am Soc Nephrol 2002; 13: 2478-87.

10. Nangaku M, Miyata T, Sada T, et al. Anti-hypertensive agents inhibit in vivo the formation of advanced glycation end products and improve renal damage in a type 2 diabetic nephropathy rat model. J Am Soc Nephrol 2003; 14: 1212-22.

11. Izuhara Y, Nangaku M, Inagi R, et al. Renoprotective properties of angiotensin receptor blockers beyond blood pressure lowering. J Am Soc Nephrol 2005; 16: 3631-41.

12. Fan Q, Liao J, Kobayashi M, et al. Candesartan reduced advanced glycation end-products accumulation and diminished nitro-oxidative stress in type 2 diabetic KK/Ta mice. Nephrol Dial Transplant 2004; 19: 3012-20.

13. Forbes JM, Cooper ME, Thallas V, et al. Reduction of the accumulation of advanced glycation end products by ACE inhibition in experimental diabetic nephropathy. Diabetes 2002; 51: 3274-82.

14. Sebeková K, Gazdíková K, Syrová D, et al. Effects of ramipril in nondiabetic nephropathy: improved parameters of oxidatives stress and potential modulation of advanced glycation end products. J Hum Hypertens 2003; 17: 265-70.

15. Saisho Y, Komiya N, Hirose H. Effect of valsartan, an angiotensin II receptor blocker, on markers of oxidation and glycation in Japanese type 2 diabetic subjects: blood pressure-independent effect of valsartan. Diabetes Res Clin Pract 2006; 74: 201-3.

16. Yamamoto Y, Hirose H, Saito I, et al. Correlation of the adipocyte-derived protein adiponectin with insulin resistance index and serum high-density lipoprotein-cholesterol, independent of body mass index, in the Japanese population. Clin Sci (Lond) 2002; 103: 137-42. 
17. Yamamoto Y, Hirose H, Saito I, Nishikai K, Saruta T. Adiponectin, an adipocyte-derived protein, predicts future insulin resistance: two-year follow-up study in Japanese population. J Clin Endocrinol Metab 2004; 89: 87-90.

18. Seino Y, Hirose H, Saito I, Itoh H. High molecular weight multimer form adiponectin as a useful marker to evaluate insulin resistance and metabolic syndrome in Japanese men. Metabolism 2007; 56: 1493-9.

19. Nakano Y, Tajima S, Yoshimi A, et al. A novel enzyme-linked immunosorbent assay specific for high-molecular-weight adiponectin. J Lipid Res 2006; 47: 1572-82.

20. Eckerson HW, Romson J, Wyte C, La Du BN. The human serum paraoxonase polymorphism: Identification of phenotypes by their response to salts. Am J Hum Genet 1983; 35: 214-27.

21. Ichihara A, Kaneshiro Y, Takemitsu T, Sakoda M, Itoh H. Benefits of candesartan on arterial and renal damage of non-diabetic hypertensive patients treated with calcium channel blockers. Am J Nephrol 2006; 26: 462-8.

22. Ichihara A, Kaneshiro Y, Sakoda M, Takemitsu T, Itoh H. Add-on amlodipine improves arterial function and structure in hypertensive patients treated with an angiotensin receptor blocker. J Cardiovasc Pharmacol 2007; 49: 161-6.

23. Chobanian AV, Bakris GL, Black HR, et al. The Seventh Report of the Joint National Committee on Prevention, Detection, Evaluation, and Treatment of High Blood Pressure.: the JNC 7 report. JAMA 2003; 289: 2560 72.

24. Japanese Society of Hypertension. The Japanese Society of Hypertension guidelines for the management of hypertension (JSH 2004). Hypertens Res 2006; 29: S1-105.

25. Monacelli F, Poggi A, Storace D, et al. Effects of valsartan therapy on protein glycoxidation. Metabolism 2006; 55: 1619-24.

26. Wang B, Pan J, Wang L, Zhu H, Yu R, Zou Y. Associations of plasma 8-isoprostane levels with the presence and extent of coronary stenosis in patients with coronary artery disease. Atherosclerosis 2006; 184: 425-30.

27. Mueller T, Dieplinger B, Gegenhuber A, et al. Serum total 8-iso-prostaglandin F2alpha: a new and independent predictor of peripheral arterial disease. J Vasc Surg 2004; 40: 768-73.

28. Kals J, Kampus P, Kals M, et al. Impact of oxidative stress on arterial elasticity in patients with atherosclerosis. Am J Hypertens 2006; 19: 902-8. 